\title{
Núcleo de Estudos da Biodiversidade da Amazônia Mato-Grossense (NEBAM): 12 anos disseminando informações científicas e democratizando o conhecimento
}

Nucleus for Study of Mato Grosso Amazon Biodiversity (NEBAM): 12 years disseminating scientific information and democrating knowledge

Nuclea de Estudios de Biodiversidad Amazónica de Mato Grosso (NEBAM): 12 años diseminando información científica y democratizando el conocimiento

Marliton Rocha Barreto ${ }^{1}$ Larissa Cavalheiro

${ }^{1}$ Biólogo. Professor Associado da Universidade Federal de Mato Grosso (UFMT). E-mail: mrb.ufmt@gmail.com, Orcid: http://orcid.org/0000-0003-3793-8855

${ }^{2}$ Bióloga. Professora Adjunta da Universidade Federal de Mato Grosso (UFMT). E-mail: larissacavalheiro@gmail.com, Orcid: http://orcid.org/0000-0002-0655-4085 
Resumo: O norte de Mato Grosso vem sofrendo perdas significativas de suas coberturas vegetais naturais; contexto aliado à falta de uma política ambiental atuante, além do desconhecimento da sociedade sobre a importância da conservação e utilização sustentável dos recursos naturais. Em 2007, os professores biólogos da Universidade Federal de Mato Grosso (UFMT) criaram o Núcleo de Estudos da Biodiversidade da Amazônia Mato-Grossense (NEBAM), objetivando, principalmente, estudos botânicos e zoológicos relacionados à riqueza, diversidade e distribuição de espécies amazônicas e do ambiente de transição Cerrado-Amazônia. Por gerar suas atividades em uma região submetida à intensa pressão antrópica, seus membros têm tomado essa diretriz como fundamento para seu desenvolvimento e, consequentemente, contribuição para o conhecimento regional. Ademais, participam, em especial, na formação de recursos humanos e, a partir de projetos de extensão, buscam contribuir para o aumento da conscientização ambiental, disseminando este conhecimento acumulado, visando à socialização e interação com a comunidade.

Palavras-chave: Amazônia; biodiversidade; Cerrado; grupo de pesquisa; Mato Grosso.

\begin{abstract}
The northern of Mato Grosso has been suffering significant losses from its natural vegetation cover; context allied to the lack of an active environmental policy, besides the lack of knowledge of society about the importance of conservation and sustainable use of natural resources. In 2007, the professors of the Federal University of Mato Grosso (UFMT) created the Nucleus for Study of Mato Grosso Amazon Biodiversity (NEBAM), which aims at botanical and zoological studies related to the richness, diversity and distribution of Amazonian species and the Cerrado-Amazon transition environment. By generating their activities in a region subjected to intense anthropic pressure, NEBAM members have taken this guideline as a foundation for their development and, consequently, a contribution to regional knowledge. In addition, they participate in the formation of human resources and, through their extension projects, seek to contribute to the increase of environmental awareness, disseminating this accumulated knowledge, aiming at socialization and interaction with the community.
\end{abstract}

Keywords: Amazon; biodiversity; Cerrado; search group; Mato Grosso.

Resumen: El norte de Mato Grosso ha sufrido pérdidas significativas de su cubierta vegetal natural, junto con la falta de una política ambiental activa y la falta de conocimiento sobre la importancia de la conservación y el uso sostenible de los recursos naturales. En 2007, los profesores de la Universidad Federal de Mato Grosso crearon el Núcleo de Estudios de la Biodiversidad Amazónica de Mato Grosso (NEBAM), cuyo objetivo son los estudios botánicos y zoológicos relacionados con la riqueza, diversidad y distribución de especies amazónicas y el entorno de transición Cerrado-Amazonas. Al desarrollar sus actividades en una región sujeta a una intensa presión antrópica, sus miembros han tomado esta guía como base para su desarrollo y, en consecuencia, una contribución al conocimiento regional. Además, participan en la capacitación de recursos humanos y, a través de proyectos de extensión, buscan contribuir al aumento de la conciencia ambiental, difundiendo este conocimiento acumulado, con el objetivo de socializar e interactuar con la comunidad.

Palabras clave: Amazon; biodiversidad; Cerrado; grupo de investigación; Mato Grosso. 


\section{INTRODUÇÃO}

A ciência moderna é praticada em colaboração, e tal pressuposto está verbalizado nos Grupos de Pesquisa, em que equipes são organizadas com o propósito de produzir conhecimento, bem como aproximar pessoas, pensamentos e realidades que resultem no fortalecimento dos indivíduos e do grupo.

O Núcleo de Estudos da Biodiversidade da Amazônia Mato-Grossense (NEBAM) foi criado em março de 2007. É, portanto, o primeiro núcleo de pesquisa do Campus Universitário de Sinop, Mato Grosso, MT, tendo sido normalizado conforme a Resolução CONSUNI n. 04, de 16 de abril de 2010, que regulamenta a Nucleação de Atividades Acadêmicas da Universidade Federal de Mato Grosso (UFMT), e aprovado na Sessão Ordinária n. 292 do CONSUNI, realizada em 26 de outubro de 2011. Também, desde 2007, o NEBAM está cadastrado no Diretório de Grupos de Pesquisa do Conselho Nacional de Desenvolvimento Científico e Tecnológico (CNPq).

O NEBAM foi criado para realizar, como principal objetivo, atividades de pesquisa científica básica, mediante inventários biológicos (Botânica e Zoologia), estudos ecológicos relacionados à descrição da riqueza, diversidade e distribuição de espécies amazônicas e do ambiente de transição Cerrado-Amazônia, bem como estudos de bioprospecção. Também, visa ao enriquecimento das coleções científicas do Acervo Biológico da Amazônia Meridional (ABAM), por meio da incorporação de elementos da fauna (coleção zoológica), da flora (herbário) e da coleção micológica.

\section{MEMBROS}

Os oito membros iniciais do Núcleo, professores biólogos da UFMT Sinop (Aline Fernandes Pontes Pires, Claudia dos Reis, Domingos de Jesus Rodrigues, Fábio Renato Borges, Larissa Cavalheiro da Silva, Lucélia Nobre Carvalho, Marcelo Henrique Ongaro Pinheiro e Marliton Rocha Barreto), contribuíram para a formação do Núcleo com 37 projetos e 31 artigos.

Após 12 anos de atividades, o Núcleo hoje é composto por 25 membros (professores/pesquisadores) somados a mais de 160 alunos, atuantes em projetos de ensino, pesquisa e de extensão; orientações de tese, de 
dissertação, de trabalhos de iniciação científica e de conclusão de curso. Continuam publicando artigos, livros e/ou capítulos de livros, apresentam trabalhos e/ou palestras em eventos nacionais e/ou internacionais, além de alguns participarem de comitês diversos, comissões e cargos administrativos.

Durante esse período, o Núcleo teve como coordenadores os professores Marcelo Henrique Ongaro Pinheiro (2007-2009), Domingos de Jesus Rodrigues (2009-2011 e 2011-2013), Leandro Dênis Battirola (2013-2014), Rafael Soares de Arruda (2014-2015) e Marliton Rocha Barreto (2015-2017 e 2017-2019).

\section{LINHAS DE PESQUISA}

Por gerar suas atividades em uma região submetida à intensa pressão antrópica, os membros do NEBAM contribuem para as discussões sobre desenvolvimento sustentável, sugerindo práticas compatíveis com a conservação do bioma amazônico, especialmente dos conjuntos de ecossistemas encontrados no Estado de Mato Grosso, a partir das linhas de pesquisa abaixo relacionadas.

- Estudos botânicos e zoológicos relacionados à descrição da riqueza, diversidade e distribuição de espécies.

- Conservação e comportamento de animais silvestres.

- Manejo e conservação de espécies de flora, de fauna e de fungos nativos e seus ecossistemas.

- Composição florística, estrutura e funcionamento da Floresta Amazônica e do ambiente de transição Cerrado-Floresta.

- Entomologia: estratégias para o Manejo Integrado de Pragas; interações artrópodes-planta-microrganismos e Bioecologia.

- Etnoconhecimento.

\section{PRODUÇÃO CIENTÍFICA}

A divulgação dos resultados de suas atividades, alicerçadas no esforço despendido pelo próprio núcleo ou em parceria com instituições de pesquisas atuantes, culmina em publicações em periódicos científicos nacionais e internacionais de ampla circulação. O Núcleo apresenta, como publicação 
técnica, artigos, capítulos de livro, livros organizados, além de diversos trabalhos apresentados em eventos nacionais e internacionais (Tabela 1); e grande parte desse resultado se dá em função da contínua atividade realizada pelos membros do NEBAM em variados projetos e orientações.

Tabela 1 - Produção técnica do Núcleo de Estudos da Biodiversidade da Amazônia Mato-Grossense em seu período de formação (2007) e dos últimos 4 biênios.

\begin{tabular}{llcccccc}
\hline Produção & \multicolumn{1}{c}{ Categorias } & \multicolumn{5}{c}{ Período } \\
\cline { 3 - 7 } & & $\mathbf{2 0 0 7}$ & $\mathbf{2 0 1 1 - 1 3}$ & $\mathbf{2 0 1 3 - 1 5}$ & $\mathbf{2 0 1 5 - 1 7}$ & $\mathbf{2 0 1 7 - 1 9}$ \\
\hline \multirow{3}{*}{ Científica } & Artigos & 63 & 55 & 94 & 80 & 161 \\
\cline { 2 - 7 } & Capítulo de Livro & 9 & 16 & 7 & 19 & 22 \\
\cline { 2 - 7 } & Livro & 1 & 1 & 8 & 3 & 5 \\
\hline \multirow{2}{*}{ Orientação } & Iniciação científica (IC) & 9 & 29 & 36 & 39 & 69 \\
\cline { 2 - 7 } & $\begin{array}{l}\text { Trab. de conclusão de } \\
\text { curso (TCC) }\end{array}$ & 9 & 22 & 47 & 30 & 29 \\
\cline { 2 - 7 } & M.e/Dr./Pós-Doutor & 0 & 14 & 42 & 56 & 98 \\
\cline { 2 - 7 } & Outras & 16 & 25 & 36 & 31 & 36 \\
\hline \multirow{2}{*}{ Projetos } & Pesquisa & 32 & 18 & 51 & 92 & 74 \\
\cline { 2 - 7 } & Extensão & 2 & 3 & 9 & 14 & 14 \\
\hline
\end{tabular}

Fonte: Arquivo dos autores.

Referente à produção científica, Meadows (1999, p. 65)

Relaciona essa produção à qualidade e à quantidade do que é produzido, e o conhecimento possibilita o compartilhamento de novos saberes resultantes das pesquisas realizadas, proporcionando interação, visibilidade, credibilidade, reconhecimento e prestígio nas comunidades científicas.

Silveira (2012, p. 118) relata que "a produção científica divulgada em periódicos científicos é importante para a constituição da ciência no âmbito acadêmico ao tornar o artigo um poderoso veículo de disseminação da informação científica". Ele é complementado por Witter (1989, p. 29), que afirma que "a produção científica concretiza-se predominantemente sob a forma de livros, teses, dissertações, artigos publicados em revistas especializadas, podendo aparecer também como resumos em anais de 
congressos e eventos similares". Entretanto a produção científica vai além, "abrange todas as realizações inerentes à pesquisa, ensino e aplicação prática da ciência, das quais podem resultar serviços, métodos e tecnologias em uma atividade de extensão à sociedade" (MUGNAINI; CARVALHO; CAMPANATTI-ORTIZ, 2006, p. 316).

Pelas definições sobre produção científica, apresentadas por Meadows (1999) e Silveira (2012), constata-se que o NEBAM tem tomado essa diretriz como fundamento para seu desenvolvimento e, consequentemente, contribuição para o conhecimento regional, razão que corrobora a participação de seus membros em diversos segmentos (Figura 1), em especial na formação de recursos humanos.

Os dados apresentados na Figura 1 foram obtidos a partir dos relatórios de atividades apresentados à Pró-Reitoria de Pesquisa da Universidade Federal de Mato Grosso a cada 2 anos, sendo o primeiro relatório preparado em setembro de 2013, referente ao período de atividades 2011-2013.

Figura 1 - Produção técnica do Núcleo de Estudos da Biodiversidade da Amazônia Mato-Grossense em 2007 (início) e nos períodos 2011-2013, 2013-2015, 2015-2017 e 2017-2019

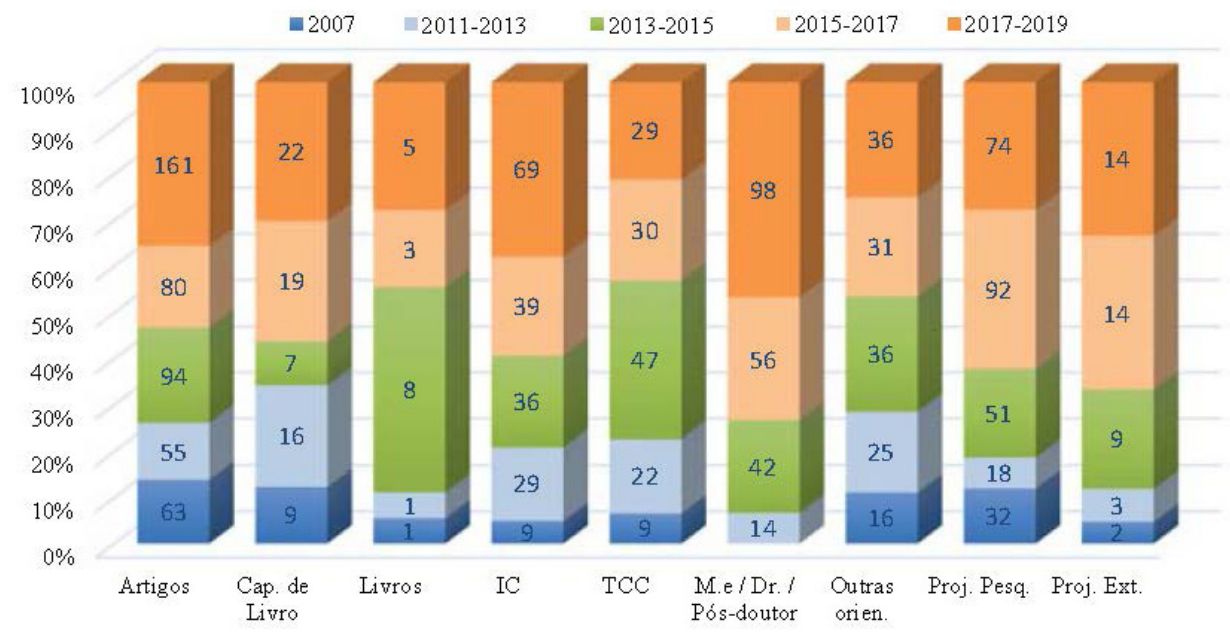

Categorias

Fonte: Arquivo dos autores. 
Conforme pode ser constatado na Tabela 1 e Figura 1, houve um salto quantitativo, com consequente avanço qualitativo, na produção dos membros do NEBAM. Independentemente do item avaliado e atendendo à proporcionalidade do número de membros por período, o Núcleo aumentou suas atividades/produção em, pelo menos, 300\% nos últimos 10 anos. É importante frisar que esse crescimento quantitativo e qualitativo do Núcleo - no ano de 2018, do total de 85 artigos publicados, 66\% pertencem aos estratos A1, A2 e B1 - é reflexo de investimentos da Universidade em contratação de professores/pesquisadores ( $90 \%$ dos atuais membros são doutores), associados a recursos provenientes de projetos individuais e/ou em parcerias, por meio de agências de fomento e/ou projetos institucionais.

O crescimento do Núcleo também está associado ao incremento da Iniciação Científica e ao início da Pós-Graduação no Campus de Sinop (2011), fator esse que permitiu aumentar significativamente a produção científica.

\section{INICIATIVA SOCIAL}

Como exemplo de iniciativa social do Núcleo, destaca-se um fórum de discussões regular hospedado pelo NEBAM, o Simpósio da Amazônia Meridional em Ciências Ambientais (SIMAMCA), desde sua terceira edição (Tabela 2). As duas primeiras edições foram de responsabilidade dos alunos e professores do curso de Ciências Biológicas da Universidade Estadual de Mato Grosso (UNEMAT), Campus Sinop.

O SIMAMCA tem por objetivo discutir sobre as diversas linhas de pesquisa nas áreas englobadas pelas Ciências Ambientais. Trata-se de um evento bianual que busca a divulgação técnico-científica no norte do Estado de Mato Grosso, englobando pesquisas multidisciplinares, envolvendo a sociedade civil, estudantes (graduação e pós-graduação) e pesquisadores de todo o país e produzindo oportunidade de criação de parcerias e inovação. O evento representa ocasião valiosa para a conscientização das instituições de pesquisas públicas e privadas no entendimento acerca da importância da Amazônia de transição e, principalmente, das diretrizes internacionais sobre o uso sustentável dos recursos naturais. 
Tabela 2 - Edições do Simpósio da Amazônia Meridional em Ciências Ambientais (SIMAMCA)

\begin{tabular}{cclc}
\hline Edição & Ano & \multicolumn{1}{c}{ Tema } & Público \\
\hline $1^{\text {a }}$ & 2006 & $\begin{array}{l}\text { Bioeconomia: Ambiente, Sociedade e } \\
\text { Tecnologia }\end{array}$ & 60 \\
\hline $2^{\text {a }}$ & 2007 & $\begin{array}{l}\text { Aquecimento Global: Sociedade e } \\
\text { Interdependência Ecossistêmica }\end{array}$ & 395 \\
\hline $3^{\text {a }}$ & 2008 & $\begin{array}{l}\text { Biodiversidade Amazônica: Conservação e } \\
\text { Sustentabilidade }\end{array}$ & 450 \\
\hline $4^{\text {a }}$ & 2010 & $\begin{array}{l}\text { Zoneamento Socioeconômico e Ecológico na } \\
\text { Amazônia: Em Busca da Sustentabilidade }\end{array}$ & 385 \\
\hline $5^{\underline{a}}$ & 2012 & $\begin{array}{l}\text { Biotecnologia e Bioprospecção a Favor da } \\
\text { Sustentabilidade }\end{array}$ & 347 \\
\hline $6^{\text {a }}$ & 2015 & $\begin{array}{l}\text { Ciência e Sociedade: Desafios para um Planeta } \\
\text { Sustentável }\end{array}$ & 388 \\
\hline $7^{\text {a }}$ & 2018 & $\begin{array}{l}\text { Amazônia de transição: Origem, } \\
\text { Desenvolvimento e Perspectivas Futuras }\end{array}$ & 457 \\
\hline
\end{tabular}

Fonte: Arquivo dos autores.

Durante o VI SIMAMCA, em 2015, esse simpósio também foi o organizador do "I Fórum de Educação Básica", que visou discutir tendências e políticas públicas voltadas ao Ensino Fundamental e Ensino Médio, bem como a formação superior e continuada de professores nas mais diversas áreas.

\section{PROJETOS DE EXTENSÃO/CURSOS DE CAPACITAÇÃO}

Para Rodrigues et al. (2013, p. 142), “a extensão surgiu com a intenção de direcionar novos caminhos para a sociedade, por meio da educação continuada e, atualmente, tornou-se um instrumento de compromisso social oportunizado pela Universidade". Ainda, segundo Saraiva (2007, p. 227):

A extensão possibilita ao acadêmico a experiência de vivências e reflexões acerca das questões da atualidade e, com base na experiência e nos conhecimentos produzidos e acumulados, o desenvolvimento de uma formação compromissada com as necessidades nacionais, regionais e locais, considerando-se a realidade brasileira.

É fato que, na sociedade atual, a informação é um elemento intrínseco ao processo de construção do conhecimento. Segundo Morin (2008, p. 15), 
é "o conhecimento vivo que conduz a grande aventura da descoberta do universo, da vida, do homem". Fica evidente, então, a relevância não só da produção da ciência, mas também sua difusão.

Com o crescente avanço técnico-científico, os problemas ambientais se intensificam em todo o planeta (DEMARCO et al., 2015, p. 101). Nesse contexto, "surge a conscientização ambiental, invocando a adoção de sistemas de gestão ambiental e o desenvolvimento de ações de responsabilidade social e ambiental, que podem se traduzir em mudanças nos modelos de gestão e na cultura organizacional" (VIEGAS; CABRAL, 2015, p. 238). Assim, o NEBAM procura, pela aplicação de seus projetos de extensão (Tabela 3), contribuir para o aumento da conscientização ambiental e, nesse sentido, as ações de extensão são valiosos instrumentos de disseminação do conhecimento acumulado, visando a sua socialização e interação com a comunidade.

Tabela 3 - Principais projetos de extensão promovidos pelo NEBAM no período 2007-2019

\section{Projeto}

1a Semana da Agronomia

A Construção do Domínio Etnozoológico "Inseto" pelos Moradores e Estudantes de Sinop, MT

A UFMT Sinop Convida: Venha nos Conhecer! Aracnologia: Sistemática, Coleta, Fixação e Gerenciamento de Dados

Capacitação de Recursos Humanos na Amazônia Meridional: Interação Ensino, Pesquisa e Extensão

Capacitação de Recursos Humanos para Pesquisas

Ecológicas de Longa Duração em Parcelas

Permanentes na Amazônia Meridional

Contribuição da Liteira Fina para o Fluxo de Carbono e Nutrientes para o Solo em Florestas Tropicais

Curso de llustração Científica

Disciplina de Campo: Biologia Floral

Etnobotânica em Sinop: Uso de Recursos Vegetais em Quintais Urbanos

Identificação de Plantas Através de Caracteres Vegetativos
Ano de Execução/

Público Atingido

2011 (65)

2009 (150), 2010 (200)

2011 (350), 2012 (700)

$2011(20)$

$2011(25)$

$2010(35)$

$2011(15)$

$2015(15)$

(2010)

$2012(220)$

$2012(65)$ 


\begin{tabular}{lc}
\hline \multicolumn{1}{c}{ Projeto } & $\begin{array}{c}\text { Ano de Execução/ } \\
\text { Público Atingido }\end{array}$ \\
\hline Introdução ao Programa ArcGIS & 2011 (15) \\
\hline Introdução ao Programa Estatístico R & $2009(30)$ \\
\hline Jardim Experimental & $2010(50)$ \\
\hline Métodos de Coletas Botânicas e Identificação em & $2010(25), 2011(30)$, \\
Parcelas Permanentes na Amazônia Meridional & $2013(40)$ \\
\hline Métodos de Medição da Estrutura da Vegetação em & $2009(30)$ \\
Parcelas Permanentes & $2012(300), 2013$ \\
\hline Museu Itinerante da Flora e da Fauna da Amazônia & $(2000), 2014(1500)$, \\
Mato-Grossense & $2017(550) 2018$ \\
& $(2000), 2019(4567)$ \\
\hline Programação Neurolinguística (PNL) & $2012(30)$ \\
\hline Seminários de Divulgação Científica & $2009(100), 2010(85)$, \\
\hline Simpósio Mato-Grossense da Amazônia Meridional & $2011(65), 2012(55)$ \\
em Ciências Ambientais (SIMAMCA) & $2012(347), 2010(385)$, \\
\hline Treinamento em Identificação Taxonômica & $2018(457)$ \\
\hline
\end{tabular}

Fonte: Arquivo dos autores.

Entre os diversos projetos de extensão realizados pelo Núcleo, o Museu Itinerante da Fauna e Flora da Amazônia Mato-Grossense está em atividade desde 2012, replicando anualmente suas atividades. Esse projeto visa à educação ambiental e ao despertar do interesse pelo meio ambiente de crianças e adolescentes de escolas da rede pública e privada de ensino de Sinop e região.

Para tanto, são realizadas apresentações e exposições da flora e fauna regionais, espécimes conservados ou vivos, na maioria das vezes com visitas à UFMT, mas também com atividades externas desenvolvidas em algumas escolas da região, no Parque Florestal de Sinop e em algumas comunidades rurais, sensibilizando os participantes sobre a importância de sua conservação.

Nas visitas, sempre pelo método de turnê-guiada, alunos de graduação, pós-graduação e professores explicam oralmente sobre os principais 
grupos da flora e da fauna, auxiliados pela exposição de materiais do herbário, insetos, aracnídeos, peixes, répteis, anfíbios e mamíferos, além de fungos. Essas apresentações são dinâmicas, possibilitando que os visitantes façam perguntas, manuseiem os organismos vivos ou conservados e, ainda, utilizem estereomicroscópio para visualização de estruturas, sempre com auxílio de um supervisor.

Entre os resultados obtidos nesse projeto, constam a consolidação e a articulação com a sociedade, além da contribuição para o desenvolvimento regional e da possibilidade de difusão de conhecimento para o público em geral, atuação que o torna pioneiro na divulgação da flora e fauna regional.

\section{ACERVO BIOLÓGICO}

Um dos maiores problemas para o homem na atualidade é o fato de que a destruição do meio ambiente acontece mais rápido que o inventário sobre as espécies de animais e plantas e os ecossistemas onde vivem. Além do óbvio empobrecimento da natureza pela extinção de espécies, a perda da biodiversidade traz um prejuízo incalculável para a humanidade, pois esse conjunto de organismos guarda muitas soluções naturais para problemas econômicos e de saúde, a maioria ainda desconhecida.

No Brasil, país de maior biodiversidade mundial, a ampliação do conhecimento sobre sua diversidade vegetal e animal fundamenta-se totalmente em coletas para herbário e coleções zoológicas. Desta forma, essas atividades não só devem ser mantidas como devem ser fortemente incrementadas, pois há evidências da existência de uma gama enorme de espécies ainda não descritas e/ou desconhecidas pela ciência.

O NEBAM também atua na criação e conservação da Coleção Zoológica do Acervo da UFMT, Campus Sinop, que tem como grupos-chave as coleções Herpetológica, Ictiológica, Entomológica e de Arthropoda não Insecta (Arachnida e Myriapoda). Essa coleção representa importante meio para o conhecimento da diversidade zoológica da Amazônia Mato-Grossense, pois é a única coleção representativa desse bioma em Mato Grosso. As demais coleções estão estabelecidas nos biomas Cerrado e Amazônia, também na Universidade Federal de Mato Grosso (Cuiabá) e na Universidade do 
Estado de Mato Grosso (Cáceres), além de coleções específicas associadas aos departamentos dessas instituições, sendo representativas para poucos grupos taxonômicos.

O Herbário CNMT também faz parte do acervo e tem como missão "Registrar a flora de Mato Grosso, em especial na transição Cerrado-Amazônia". É igualmente importante para a pesquisa de base (taxonomia e florística), bem como para pesquisas avançadas (Ecologia, Biogeografia, Bioquímica, Bioprospecção). O Herbário CNMT realiza intercâmbios de material com diversas instituições nacionais, atua como ponto fundamental nas atividades de ensino de graduação e pós-graduação, além de atividades de extensão. Ademais, tem vinculadas a ele a coleção de fungos microscópicos, sob a sigla CNMTf, e uma carpoteca em via seca e úmida.

\section{INTERAÇÕES COM OUTRAS INSTITUIÇÕES}

Sem dúvida, visando ao fortalecimento do grupo e à continuidade das atividades, o NEBAM vem desde o início realizando parcerias com Instituições de Pesquisa (EMBRAPA - Amapá, Agrossilvipastoril, Floresta; Herbário Virtual da Flora e dos Fungos [INCT/HVFF]; Instituto de Botânica de São Paulo; Instituto Federal [IFMT Cáceres]; Instituto Florestal do Estado de São Paulo; Instituto Nacional de Pesquisas da Amazônia [INPA]; Michigan University, EUA; Museu de História Natural Basel, Suíça; Prefeitura de Sinop; Secretaria de Estado de Meio Ambiente de MT; Universidade Estadual de Londrina; Universidade Estadual de Mato Grosso [UNEMAT Alta Floresta, Cáceres, Sinop]; Agropel Sementes; Empresa Brandt Soluções em Agricultura Ltda; Stoller do Brasil; Agrotechnica Indústria e Comércio de Fertilizantes Ltda; Dow AgroSciences; BASF; Universidade Federal de Santa Catarina; Universidade Federal do Rio Grande do Norte; entre outros) - e/ou com pesquisadores individualmente, que visam ao fortalecimento do grupo e à continuidade das atividades por meio de cursos de extensão e capacitação, parcerias em projetos de pesquisa e extensão, bolsa de estudos, uso de equipamentos etc. 


\section{CONSIDERAÇÕES FINAIS}

Diante do exposto, os membros do NEBAM atuam em projetos de ensino, de pesquisa e de extensão, em orientações diversas, fazeres somados à produção de artigos, de livros e/ou capítulos; ademais, apresentam trabalhos e/ou palestras em eventos científicos nacionais e/ou internacionais. Alguns membros exercem funções administrativas e atuam em diversos comitês, dentro e fora da instituição, assim como operam em organização de eventos científicos e junto à comunidade. No desempenho de suas atividades em uma região submetida à intensa pressão antrópica, os membros do NEBAM continuam contribuindo para as discussões sobre desenvolvimento sustentável, sugerindo práticas compatíveis à conservação dos ecossistemas amazônicos, especialmente aqueles encontrados no Estado de Mato Grosso.

Aliado à UFMT, o NEBAM busca ampliar sua atuação junto à sociedade mato-grossense, tendo em vista a geração de benefícios sociais efetivamente significativos. Esse entendimento traduz a necessidade de a Universidade conhecer, cada vez mais e melhor, os anseios da sociedade, pois é a adequada intervenção na realidade que caracteriza o desafio político e, sobretudo, o papel da extensão universitária, atuando de maneira harmônica, articulada e integrada com o ensino e a pesquisa.

\section{REFERÊNCIAS}

DEMARCO, J. O.; CADORE, J. S.; INSELSPERGER, V.; RODRIGUES, A. C.; FORTES, P. R. Extensão universitária na conscientização ambiental em escolas de educação básica. Revista Monografias Ambientais, Santa Maria, Edição Especial, p. 101-7, 2015. Disponível em: https://periodicos.ufsm.br/remoa/article/view/18747. Acesso em: 9 set. 2019.

MEADOWS, A. J. A comunicação científica. Brasília: Briquet de Lemos Livros, 1999.

MORIN, E. Ciência com Consciência. 12. ed. Rio de Janeiro: Bertrand Brasil, 2008.

MUGNAINI, R.; CARVALHO, T.; CAMPANATTI-ORTIZ, H. Indicadores de produção científica: uma discussão conceitual. In: POBLACION, D. A. et al. (Org.). Comunicação e produção científica: contexto e avaliação. São Paulo: Angellara, 2006. p. 313-40. 
RODRIGUES, A. L. L.; PRATA, M. S.; BATALHA, T. B. S.; COSTA, C. L. N. A.; PASSOSNETO, I. F. Contribuições da extensão universitária na sociedade. Cadernos de Graduação - Ciências Humanas e Sociais, Aracaju, v. 1, n. 16. p. 141-8, mar. 2013. Disponível em: https://periodicos.set.edu.br/index.php/cadernohumanas/article/ viewFile/494/254 . Acesso em: 9 set. 2019.

SARAIVA, J. L. Papel da extensão universitária na formação de estudantes e professores. Brasília Médica, Brasília, v. 44, n. 3, p. 220-5, 2007. Disponível em: https://rl.art.br/arquivos/3635754.doc. Acesso em: 9 set. 2019.

SILVEIRA, J. P. B. A produção científica em periódicos institucionais: um estudo da revista Biblos. Encontros Bibli - Revista Eletrônica de Biblioteconomia e Ciência da Informação, Florianópolis, v. 17, n. 33, p. 116-33, jan./abr. 2012. Disponível em: http://dx.doi.org/10.5007/1518-2924.2012v17n33p116. Acesso em: 9 set. 2019.

VIEGAS, S. F. S.; CABRAL, E. R. Práticas de Sustentabilidade em Instituições de Ensino Superior: Evidências de Mudanças na Gestão Organizacional. Gual, Florianópolis, v. 8, n. 1, p. 236-59, jan. 2015. Disponível em: https://periodicos.ufsc.br/index.php/ gual/article/view/1983-4535.2015v8n1p236. Acesso em: 9 set. 2019.

WITTER, G. P. Pós-graduação e produção científica: a questão de autoria. TransInformação, v. 1, n. 1, p. 29-37, 1989. Disponível em: http://periodicos.puccampinas.edu.br/seer/index.php/transinfo/article/view/1698. Acesso em: 9 set. 2019. 